\title{
Phase behavior of hard spheres with a short-range Yukawa attraction
}

\author{
Marjolein Dijkstra \\ Debye Institute, Soft Condensed Matter Physics, Utrecht University, Princetonplein 5, 3584 CC Utrecht, The Netherlands
}

(Received 29 April 2002; published 14 August 2002)

\begin{abstract}
The phase diagram of a system consisting of hard spheres with an attractive Yukawa interaction is computed by Monte Carlo simulations. Upon decreasing the range of attraction, we find the following scenarios: (a) a stable fluid-fluid and fluid-solid transition, a triple point with fluid-fluid-solid coexistence, and a metastable isostructural solid-solid transition (b) a fluid-fluid and isostructural solid-solid transition, which are both metastable with respect to a broad fluid-solid transition, (c) a metastable fluid-fluid transition, a stable isostructural solid-solid and fluid-solid transition and a triple point with fluid-solid-solid coexistence. Our results show that a large part of the fluid-solid coexistence region is occupied by metastable fluid-fluid and/or solid-solid coexistence regions, which has repercussions on the kinetics and on the nonequilibrium behavior of the system, such as nucleation, vitrification, and gelation.
\end{abstract}

DOI: 10.1103/PhysRevE.66.021402

PACS number(s): 82.70.Dd, 61.20.Gy

\section{INTRODUCTION}

Since van der Waals it is known that the occurrence of a vapor-liquid transition in the phase diagram of simple fluids is strongly related to the presence of long-range attractive interactions between the particles. Using a simple mean-field theory and assuming long-range attractions and short-range repulsions for the molecules, van der Waals showed the existence of a critical temperature, below which the homogeneous fluid demixes into a vapor and liquid phase. Above this critical temperature, no liquid-vapor transition takes place. Moreover, the theory predicts a vanishing critical temperature if there are no attractions between the particles. This implies that any non zero temperature is above the critical temperature in a purely repulsive system, and no vaporliquid coexistence appears in the phase diagram. More recently, this relationship between attractive interactions and the existence of a stable vapor-liquid transition was refined substantially. It was shown by computer simulations $[1,2]$, density functional calculations [3-5], and integral equation theories [6] that the range of the attractive interactions determines whether or not a given substance can have a stable vapor-liquid coexistence. The range of the attractions cannot be changed for simple liquids, e.g., argon, but we can tune the attractions in colloidal systems by adding smaller components, such as nonadsorbing polymer or colloidal particles. The minimum range of the attractions required for a stable liquid-vapor coexistence is about one sixth of the range of the repulsions. For shorter-range attractions, the liquid-vapor coexistence region becomes metastable with respect to the freezing transition and when the range of attractions is smaller than about one twentieth of the range of the repulsions, a stable isostructural solid-solid transition [2-5,7-10] appears in the phase diagram. It is already noted in Refs. [3-5] that a spinodal instability can arise in both the fluid and the solid phase. The competition between both instabilities and the freezing transition can produce three different types of phase diagrams: (i) a stable fluid-fluid and fluidsolid coexistence and, a fluid-fluid-solid triple point, (ii) only fluid-solid coexistence, and (iii) a stable solid-solid and fluid-solid coexistence and, a fluid-solid-solid triple point
[3-5]. However, most studies that investigate the relation between the range of attraction of the intermolecular potential and the stability of the fluid-fluid phase separation or the isostructural solid-solid transition are focused on the equilibrium phase behavior of the system and ignore the presence of metastable fluid-fluid or solid-solid coexistence regions. However, the appearance of metastable regions in the phase diagram is important as it influences the phase kinetics [11] and the nonequilibrium behavior of the system, such as nucleation, vitrification, or gelation. For instance, experiments on binary mixtures of colloidal spheres and on colloidpolymer mixtures show that there is a link between non equilibrium states and the presence of metastable coexistence regions in the phase diagram $[12,13]$. Moreover, recent experiments show that crystallization of spherical proteins only occurs under certain conditions [14] and a computer simulation study shows that the presence of a metastable critical point enhances protein crystallization [15]. In addition, these metastable regions are also important when the crystalline phase is destabilized by, for instance, small amounts of polydispersity of the colloids or by applying a constraint that stabilizes the fluid phase, which would otherwise be metastable [16].

In this paper, we report a numerical study of the full phase behavior, including the metastable coexistence regions, of hard spheres with a short-range attractive Yukawa interaction. We show that in all phase diagrams, spinodal instabilities are observed in both the solid and the fluid phase, resulting in a (meta) stable isostructural solid-solid and a fluidfluid phase separation. However, only one of those instabilities can lead to a stable phase separation. We never find simultaneously, a stable isostructural solid-solid and a stable fluid-fluid coexistence in the same phase diagram, which is in agreement with previous studies [2-5,7].

In order to map out the phase diagram of the hard-core attractive Yukawa system, we calculate the Helmholtz free energy as a function of the density using thermodynamic integration [17]. In the following section, we briefly describe the simulation method. We present and discuss the phase diagrams of the hard-core attractive Yukawa system in Sec. III and we end with some conclusions in Sec. IV. 


\section{SIMULATIONS}

We consider a system of hard spheres with diameter $\sigma$ interacting with an attractive Yukawa interaction,

$$
\phi(r)= \begin{cases}\infty, & r<\sigma \\ -\epsilon \frac{\exp [\kappa \sigma(1-r / \sigma)]}{r / \sigma}, & r \geqslant \sigma,\end{cases}
$$

where $\epsilon$ denotes the well depth, and $\kappa^{-1}$ the Yukawa screening length. In order to determine the phase diagram of a system of hard spheres interacting with an attractive Yukawa interaction, we first calculate the Helmholtz free energy $F$, as a function of $N, V$, and the reduced temperature $T^{*}=k_{B} T / \epsilon$ with $k_{B}$ Boltzmann's constant. We actually measure the dimensionless free energy density $f=(\pi / 6) \sigma^{3} F / V$ as a function of $T^{*}$ and $\eta=\pi \sigma^{3} N / 6 \mathrm{~V}$. As the free energy cannot be measured directly in a Monte Carlo (MC) simulation, we use thermodynamic integration to relate the free energy of the system of interest to that of a reference hard-sphere system at the same packing fraction $\eta$. To this end we introduce the auxiliary Hamiltonian

$$
H_{\lambda}=\sum_{i<j}^{N}\left[\phi_{h s}\left(r_{i j}\right)+\lambda \phi_{y u k}\left(r_{i j}\right)\right]
$$

with

$$
\phi_{h s}(r)= \begin{cases}\infty, & r<\sigma \\ 0, & r \geqslant \sigma\end{cases}
$$

and

$$
\phi_{y u k}(r)= \begin{cases}0, & r<\sigma \\ -\epsilon \frac{\exp [\kappa \sigma(1-r / \sigma)]}{r / \sigma}, & r \geqslant \sigma,\end{cases}
$$

and where $0 \leqslant \lambda \leqslant 1$ is a dimensionless coupling parameter: at $\lambda=0$ the auxiliary Hamiltonian is that of the pure system of $N$ hard spheres, while at $\lambda=1$ it is the Hamiltonian of interest. It is a standard result $[1,2,7,18]$ that the Helmholtz free energy of the hard-sphere attractive Yukawa system reads

$$
F\left(N, V, T^{*}\right)=F_{h s}(N, V)+\int_{0}^{1} d \lambda\left\langle\sum_{i<j}^{N} \phi_{y u k}\left(r_{i j}\right)\right\rangle_{N, V, T^{*}, \lambda},
$$

where $F_{h s}(N, V)$ is the free energy of the pure reference system of $N$ hard spheres in a volume $V(\lambda=0)$, for which we use the Carnahan-Starling expressions [19] for the fluid, and the analytic form for the equation of state proposed by Hall [20] for the solid phase. In the latter case an integration constant is determined, such that the known fluid-solid coexistence of the pure hard-sphere system is recovered [21]. The angular brackets $\langle\cdots\rangle_{N, V, T^{*}, \lambda}$ denote a canonical average over a system of $N$ particles in a volume $V$ and at temperature $T^{*}$ interacting via the auxiliary Hamiltonian $H_{\lambda}$. The integrand in Eq. (5) can, for a fixed $\lambda$, be measured in a standard MC calculation; for the numerical $\lambda$ integration we use a 10-point Gauss-Legendre quadrature [22].

In order to map out the phase diagram, $f\left(\eta, T^{*}\right)$ must be determined from $\lambda$ integrations for many state points $\left(\eta, T^{*}\right)$. We therefore chose to simulate relatively small systems, with $N=108$. It is worth noting that for such a small system size, the formation of one or more new phases is prevented as it costs too much free energy to form an interface. We were therefore able to simulate the metastable phases for temperatures as low as $T^{*}=0.1$. In order to construct the full phase diagram we employ common tangent constructions at fixed $T^{*}$ to obtain the coexisting phases. We fitted polynomials to $f$ and computed the pressure and chemical potential at each $\eta$. The densities of the coexisting phases can then be determined by equating the pressures and chemical potentials in both phases.

\section{RESULTS AND DISCUSSION}

The above procedure has been carried out to determine $f\left(\eta, T^{*}\right)$ for the fluid and the solid phase for $\kappa \sigma$ $=3.9,7,25$, and 100. At sufficiently low $T^{*}$, we find spinodal instabilities in the fluid and the solid free energy density curve. Employing a common tangent construction on the fluid free energy density curve versus $\eta$, one finds at the points of tangency, the low-density fluid phase, which coexists with the high-density fluid phase. Carrying out the same procedure on the solid free energy density curve versus $\eta$, we find coexistence between a low-density (expanded) facecentered-cubic (fcc) solid and a high-density (compressed) fcc solid. However, one can always perform a common tangent construction between the free energy density curve of the fluid phase and that of the solid and one should check whether the fluid-fluid and solid-solid transitions are (meta) stable with respect to the fluid-solid transition. Figures 1-4 show the resulting phase diagrams in the $\left(\eta, T^{*}\right)$ plane. The shaded areas represent the (metastable) fluid-fluid and solidsolid two-phase regions. It is worth noting that we find spinodal instabilities in both the fluid phase as well as in the solid phase in all the phase diagrams considered here.

For $\kappa \sigma=3.9$, we observe in Fig. 1 a stable fluid-fluid coexistence, a stable fluid-solid coexistence, a triple point with the fluid-fluid-solid coexistence, and a metastable isostructural solid-solid coexistence.

For shorter-ranged Yukawa potentials, i.e., $\kappa \sigma=7$ and 25 , we observe in Figs. 2 and 3 that both the fluid-fluid and the solid-solid coexistence regions are metastable with respect to freezing. At sufficiently low $T^{*}$, we find an enormous widening of the fluid-solid transition. This implies that the coexisting fluid and solid phase become progressively more dilute and dense, respectively, upon decreasing $T^{*}$. This widening is consistent with findings by Gast et al. [23] in perturbation theory studies of colloid-polymer mixtures using the Asakura-Oosawa depletion potential $[24,25]$ and with computer simulations of binary hard-sphere mixtures using the depletion potential picture $[26,27]$. The shape of the coexistence curve for $T^{*}<0.3$ implies that the fluid phase only persists to very low values of $\eta$.

For $\kappa \sigma=100$, a stable isostructural solid-solid coexist- 


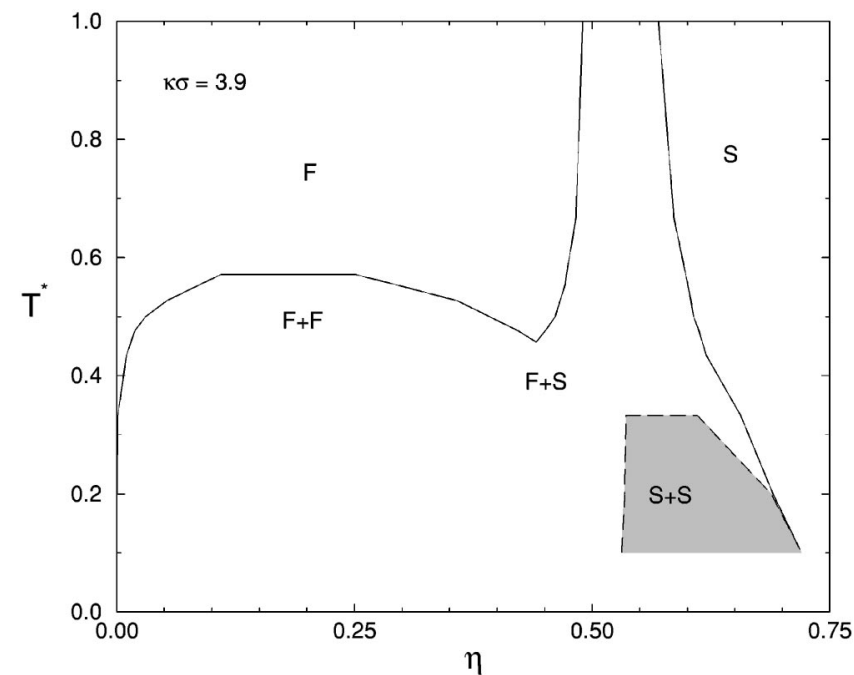

FIG. 1. Phase diagram of a system of hard spheres interacting with a Yukawa attraction with $\kappa \sigma=3.9$ in the $T^{*}-\eta$ plane, with $T^{*}=k_{B} T / \epsilon$ the reduced temperature and $\eta=\pi \sigma^{3} N / 6 V$ the packing fraction. $F$ and $S$ denote the stable fluid and solid (fcc) phase. $F$ $+S, F+F$, and $S+S$ denote, respectively, the stable fluid-solid, the stable fluid-fluid, and the metastable solid-solid coexistence regions.

ence is observed in Fig. 4, while the fluid-fluid coexistence is metastable with respect to freezing. In addition, we find a triple point with the fluid-solid-solid coexistence.

In summary, we have shown that varying the range of the attraction in a system of particles interacting with a hardsphere Yukawa attraction leads to three distinct phase diagrams: (i) a stable fluid-fluid and fluid-solid transition, a triple point with fluid-fluid-solid coexistence, and a meta-

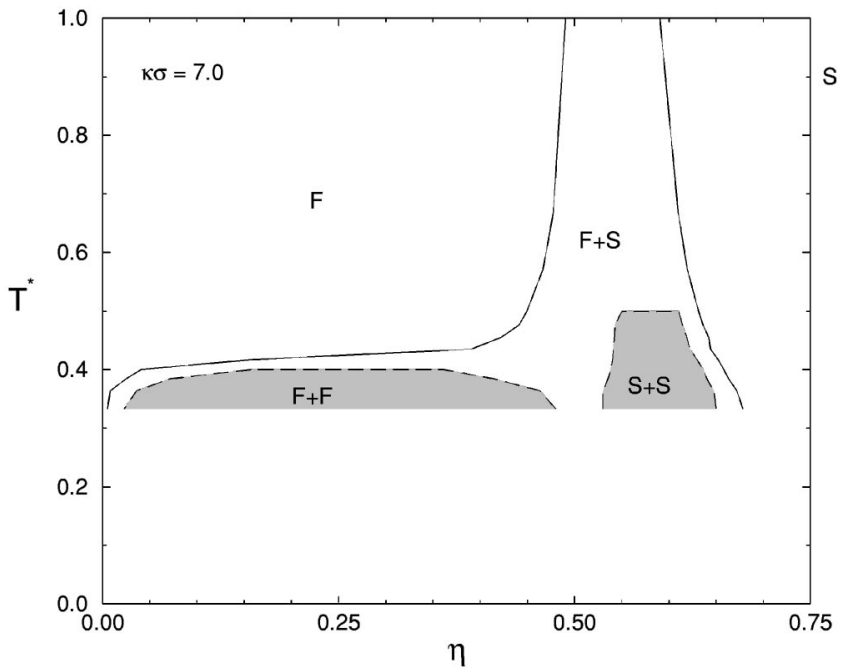

FIG. 2. Phase diagram of a system of hard spheres interacting with a Yukawa attraction with $\kappa \sigma=7$ in the $T^{*}-\eta$ plane, with $T^{*}$ $=k_{B} T / \epsilon$ the reduced temperature and $\eta=\pi \sigma^{3} N / 6 \mathrm{~V}$ the packing fraction. $F$ and $S$ denote the stable fluid and solid (fcc) phase. $F$ $+S, F+F$, and $S+S$ denote, respectively, the stable fluid-solid, the metastable fluid-fluid, and the metastable solid-solid coexistence regions.

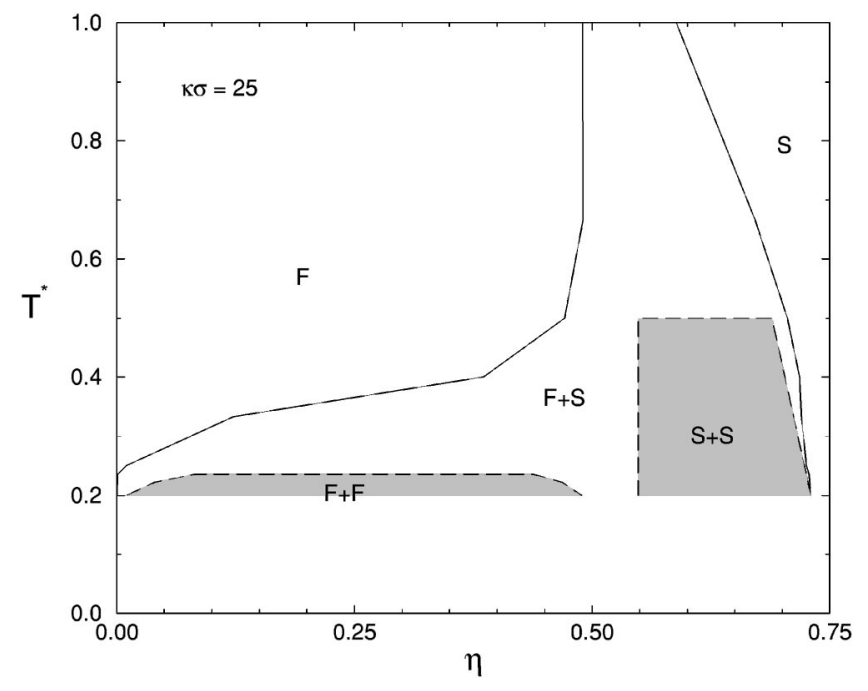

FIG. 3. Phase diagram of a system of hard spheres interacting with a Yukawa attraction with $\kappa \sigma=25$ in the $T^{*}{ }_{-} \eta$ plane, with $T^{*}=k_{B} T / \epsilon$ the reduced temperature and $\eta=\pi \sigma^{3} N / 6 V$ the packing fraction. $F$ and $S$ denote the stable fluid and solid (fcc) phase. $F$ $+S, F+F$, and $S+S$ denote, respectively, the stable fluid-solid, the metastable fluid-fluid, and the metastable solid-solid coexistence regions.

stable isostructural solid-solid transition, (ii) a fluid-fluid and isostructural solid-solid transition that are both metastable with respect to a broad fluid-solid transition, and (iii) a fluidfluid transition, which is metastable with respect to a broad fluid-solid transition and a stable isostructural solid-solid transition and a triple point with fluid-solid-solid coexistence. This is in agreement with a theoretical study of Tejero et al. using a variational approach based on the Gibbs-

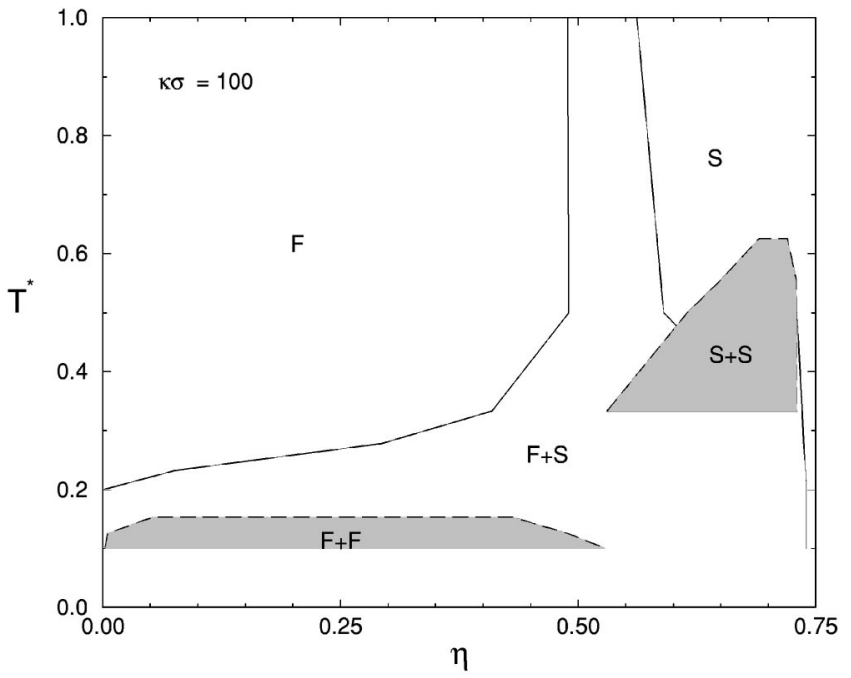

FIG. 4. Phase diagram of a system of hard spheres interacting with a Yukawa attraction with $\kappa \sigma=100$ in the $T^{*}-\eta$ plane, with $T^{*}=k_{B} T / \epsilon$ the reduced temperature and $\eta=\pi \sigma^{3} N / 6 V$ the packing fraction. $F$ and $S$ denote the stable fluid and solid (fcc) phase. $F$ $+S, F+F$, and $S+S$ denote, respectively, the stable fluid-solid, the metastable fluid-fluid, and the stable solid-solid coexistence regions. 
Bogliubov inequality for a fluid of particles interacting with a double Yukawa potential $[3,4]$, and with a study using the van der Waals approximation for the solid [5]. However, the location of the metastable regions are not shown in their phase diagrams. Our phase diagrams for $\kappa \sigma=3.9$ and 7 are also in good agreement with previous computer simulations of the hard-core attractive Yukawa system using the Gibbsensemble technique for the fluid-fluid coexistence and the Kofke integration technique for the fluid-solid coexistence [1]. However, the authors did not consider the (metastable) isostructural solid-solid coexistence in their work. In another study, Hagen and co-workers computed phase diagrams of hard spheres with shorter-ranged Yukawa attractions. For $\kappa \sigma=25$, they only show the stable fluid-solid coexistence from first-order perturbation theory using the hard-sphere fluid as a reference system. They do not show the metastable solid-solid and fluid-fluid transition. Our "exact" results for the fluid-solid phase boundaries show that the widening of the coexistence region at the solid side is less abrupt compared with the fluid-solid region obtained from first-order perturbation theory.

For $\kappa \sigma=100$, Hagen and co-workers computed the solidsolid coexistence using thermodynamic integration and we find a good agreement with their results. Again, they did not compute the fluid-solid and the metastable fluid-fluid coexistence.

Our results disagree with the findings of Sear using a simple perturbation theory about a hard-sphere fluid [28]. He shows that the critical point of the fluid-fluid transition tends towards the random-close-packing density of hard spheres, as the range of the attraction tends to zero. In our work on binary hard-sphere mixtures, we have already shown that the structure of hard spheres with a very short-range potential differ enormously with that of the reference hard-sphere fluid at the same packing fraction, i.e., much higher contact values for the pair correlation functions are found compared with the hard-sphere system. This enormous difference in the structure signals the breakdown of perturbation theory $[26,27]$.

More importantly, our results show that a large part of the fluid-solid coexistence region is occupied by metastable fluid-fluid and/or solid-solid coexistence regions, which has repercussions on the kinetics [11] and on the nonequilibrium behavior of the system, such as nucleation, vitrification, and gelation.

It is also worth noting that a metastable phase can only be observed when its lifetime is longer than the observation time and a property of the metastable phase, e.g., its free energy, can only be measured when its characteristic relaxation time is shorter than the system's lifetime [16]. Eventually, at infinite time, the system should reach the equilibrium state. In an experiment, a metastable phase can persist for a long time by careful preparation of the sample, e.g., avoiding vibrations, impurities, and irregularities on the walls that can trigger nucleation. In our simulations, we use very small system sizes and therefore the formation of a new phase is prevented as it costs too much interfacial free energy to create an interface. This enables us to simulate the metastable phases till temperatures as low as $T^{*}=0.1$, which are prob-
TABLE I. The reduced critical temperatures $T_{c}^{*}=k_{B} T_{c} / \epsilon$ of the fluid-fluid coexistence region for a hard-sphere attractive Yukawa system with varying Yukawa screening length $\kappa^{-1}$. The effective range of the attraction at the critical point is given by the critical stickiness parameter $\tau_{c}$, and $R_{c}=\lambda-1$ is the critical range of attraction of the corresponding square-well system.

\begin{tabular}{cccc}
\hline \hline$\kappa$ & $T_{c}^{*}$ & $\tau_{c}$ & $R_{c}$ \\
\hline 3.9 & 0.5714 & 0.0973 & 0.1549 \\
7 & 0.4000 & 0.1010 & 0.0689 \\
25 & 0.2353 & 0.1060 & 0.0113 \\
100 & 0.1538 & 0.1078 & 0.0012 \\
\hline \hline
\end{tabular}

ably inaccessible experimentally.

Finally, it is shown in Ref. [29] that the reduced second virial coefficient is a convenient measure for the effective range of the attractive part of the potential. The reduced second virial coefficient can be calculated once the functional form of the pair potential $\phi(r)$ is known,

$$
B_{2}^{*}=\frac{3}{\sigma_{e f f}^{3}} \int_{0}^{\infty} d r r^{2}\left[1-e^{-\phi(r) / k_{B} T}\right]
$$

where $\sigma_{e f f}$ is the effective hard-core diameter. For hard spheres with diameter $\sigma$ which interact with short-range attractions, the effective hard-core diameter is given by $\sigma_{e f f}$ $=\sigma$ and the reduced second virial coefficient is often expressed in terms of a parameter $\tau$,

$$
B_{2}^{*}=1-\frac{1}{4 \tau}
$$

References $[29,30]$ show that the reduced second virial coefficient and stickiness parameter $\tau$ are remarkably constant, i.e., $\tau \simeq 0.1$, at the critical point of the fluid-fluid transition for different functional forms of the pair potentials. In Table I, we show the critical reduced temperatures $T_{c}^{*}=k_{B} T_{c} / \epsilon$ of the fluid-fluid transition and the corresponding value of $\tau_{c}$ for varying $\kappa$. We find that the values of $\tau_{c}$ at the critical point are indeed about 0.1. As mentioned in Ref. [29], we can now estimate the relative location of the fluid-fluid coexistence and solid-fluid coexistence, by mapping the system of interest onto the square-well system that yields the same value for $\tau$ at the same reduced temperature $T^{*}$. The pair potential of the square-well system is given by

$$
\phi(r)= \begin{cases}\infty, & r<\sigma \\ -\epsilon, & \sigma<r \geqslant \lambda \sigma \\ 0, & r>\lambda \sigma .\end{cases}
$$

We also give the critical range $R_{c}=\lambda-1$ of the corresponding square-well system in Table I. Reference [29] shows that the fluid-fluid transition is stable with respect to freezing when $R_{c} \geqslant 0.14$ at the critical point, and becomes metastable for $R_{c}<0.14$. We now compare our phase diagrams with the range of attraction $R_{c}$ of the equivalent square-well system listed in Table I. We indeed find for $\kappa=3.9$, which yields a 
stable fluid-fluid transition in Fig. 1, that the range of attraction $R_{c}$ is larger than 0.14 . For $\kappa=7,25$, and 100 , we find $R_{c}<0.14$ and only metastable fluid-fluid transitions are observed in Figs. 2-4.

\section{CONCLUSION}

In conclusion, we have determined the full phase behavior, including the metastable coexistence regions, of hard spheres with a short-range attractive Yukawa interaction using computer simulations. We show that instabilities can be obtained in both the solid and the fluid phase, resulting in a (meta) stable isostructural solid-solid and a fluid-fluid phase separation. However, only one of those instabilities can lead to a stable phase separation and we never find a stable isostructural solid-solid as well as a stable fluid-fluid coexistence in the same phase diagram. The focus of this paper is on the location of the metastable coexistence regions. We find that large portions of the fluid-solid coexistence are occupied by metastable fluid-fluid or solid-solid coexistence regions, which has consequences for the phase kinetics [11] and non equilibrium behavior of the system.

\section{ACKNOWLEDGMENT}

M.D. is grateful to FOM for financial support of a workstation under the FOM/V stimulerings program.
[1] M.H.J. Hagen and D. Frenkel, J. Chem. Phys. 101, 4093 (1994).

[2] P. Bolhuis, M. Hagen, and D. Frenkel, Phys. Rev. E 50, 4880 (1994).

[3] C.F. Tejero, A. Daanoun, H.N.W. Lekkerkerker, and M. Baus, Phys. Rev. Lett. 73, 752 (1994).

[4] C.F. Tejero, A. Daanoun, H.N.W. Lekkerkerker, and M. Baus, Phys. Rev. E 51, 558 (1995).

[5] A. Daanoun, C.F. Tejero, and M. Baus, Phys. Rev. E 50, 2913 (1994).

[6] C. Caccamo, G. Pellicane, D. Costa, D. Pini, and G. Stell, Phys. Rev. E 60, 5533 (1999).

[7] P. Bolhuis and D. Frenkel, Phys. Rev. Lett. 72, 2211 (1994).

[8] C.N. Likos and G. Senatore, J. Phys.: Condens. Matter 7, 6797 (1995).

[9] Zs.T. Németh and C.N. Likos, J. Phys.: Condens. Matter 7, L537 (1995).

[10] C.N. Likos, Zs.T. Németh, and H. Löwen, J. Phys.: Condens. Matter 6, 10965 (1994).

[11] F. Renth, W.C.K. Poon, and R.M.L. Evans, Phys. Rev. E 64, 031402 (2001); R.M.L. Evans, W.C.K. Poon, and F. Renth, ibid. 64, 031403 (2001).

[12] W.C.K. Poon, A.D. Pirie, M.D. Haw, and P.N. Pusey, Physica A 235, 110 (1997); R.M.L. Evans and W.C.K. Poon, Phys. Rev. E 56, 5748 (1997).

[13] A. Imhof and J.K.G. Dhont, Phys. Rev. Lett. 75, 1662 (1995); Phys. Rev. E 52, 6344 (1995).
[14] D. Rosenbaum, P.C. Zamora, and C.F. Zukoski, Phys. Rev. Lett. 76, 150 (1996).

[15] P.R. ten Wolde and D. Frenkel, Science 277, 1975 (1997).

[16] P.G. Debenedetti, in Metastable Liquids (Princeton University Press, Princeton, NJ, 1996).

[17] D. Frenkel and B. Smit, in Understanding Molecular Simulation: From Algorithms to Applications (Academic, New York, 1996).

[18] J.P. Hansen and L. Verlet, Phys. Rev. 184, 151 (1969).

[19] N.F. Carnahan and K.E. Starling, J. Chem. Phys. 51, 635 (1969).

[20] R. Hall, J. Chem. Phys. 57, 2252 (1972).

[21] W.G. Hoover and F.H. Ree, J. Chem. Phys. 49, 3609 (1968).

[22] W.H. Press, B.P. Flannery, S.A. Teukolsky, and W.T. Vetterling, in Numerical Recipes in Fortran (Cambridge University Press, Cambridge, England, 1992).

[23] A.P. Gast, C.K. Hall, and W.B. Russel, J. Colloid Interface Sci. 96, 251 (1983).

[24] S. Asakura and F. Oosawa, J. Chem. Phys. 22, 1255 (1954).

[25] A. Vrij, Pure Appl. Chem. 48, 471 (1976).

[26] M. Dijkstra, R. van Roij, and R. Evans, Phys. Rev. Lett. 81, 2268 (1998).

[27] M. Dijkstra, R. van Roij, and R. Evans, Phys. Rev. E 59, 5744 (1999).

[28] R.P. Sear, Phys. Rev. E 61, 6019 (2000).

[29] M.G. Noro and D. Frenkel, J. Chem. Phys. 113, 2941 (2000).

[30] G.A. Vliegenthart and H.N.W. Lekkerkerker, J. Chem. Phys. 112, 5364 (2000). 